\title{
Intravenous gammaglobulin therapy in the thrombocytopenia of haemorrhagic varicella
}

\author{
D.M. Large, A. Basu and H. O'Brien' \\ Department of Medicine and 'Department of Haematology, Cumberland Infirmary, Carlisle CA2 7HY, \\ $U K$
}

\begin{abstract}
Summary: We report the case of a teenage boy with thrombocytopenia complicating varicella infection, which responded promptly to treatment with intravenous immunoglobulin therapy. Such treatment is well recognized in the management of immune thrombocytopenia, particularly where steroids are contra-indicated, but we have not found any previous case reports of the use of immunoglobulin in this condition.
\end{abstract}

\section{Introduction}

Varicella infection is usually a benign and selflimiting condition in childhood and adolescence; thrombocytopenia is an uncommon complication. Fatal haemorrhage has, however, been reported. ${ }^{1}$ We describe a case of varicella-induced thrombocytopenia in a 17 year old boy, which responded to treatment with intravenous gammagobulin.

\section{Case report}

A previously fit 17 year old boy developed an extensive rash of varicella vesicles with typical distribution. On the 8th day he awoke to find his bed-linen covered with blood spots and noted the presence of fresh haemorrhagic lesions over the oral mucous membranes and the inner eyelids within the conjunctival sac. Most of the vesicles on his body were haemorrhagic. There was no epistaxis, haemoptysis or haematuria and he did not experience headaches or visual symptoms. He was admitted to hospital 2 days later. Examination showed no bruising or fundal haemorrhage. Results of investigations showed haemoglobin $15.6 \mathrm{~g} / \mathrm{dl}$, white cell count $13.5 \times 10^{9} / 1$ (normal differential count), platelet count $5.0 \times 10^{9} / 1$; clotting screen, in particular prothrombin time, was normal. Bone marrow examination showed increased megakaryocytes, but was otherwise normal. Chest X-ray was unremarkable. Subsequent investigations showed normal levels of urea, creatinine, electrolytes, liver enzymes, immuno-

Correspondence: D.M. Large, M.D., F.R.C.P. Accepted: 25 October 1990 globulins IgA, IgE and IgM, anti-nuclear factor, complement $\mathrm{C} 3, \mathrm{C} 4$ and $\mathrm{CH} 100$. The next morning platelets were absent on the blood film.

Treatment was commenced with intravenous gammaglobulin (sandoglobulin) $0.4 \mathrm{~g} / \mathrm{kg}(24 \mathrm{~g})$ daily; because of the long distance from the Regional Blood Transfusion Service platelets were ordered and subsequently transfused, in view of the complete absence of platelets on the film. The response to treatment and the apparently increasing survival of transfused platelets on days 2 and 3 , is shown in Figure 1. Platelet counts were checked

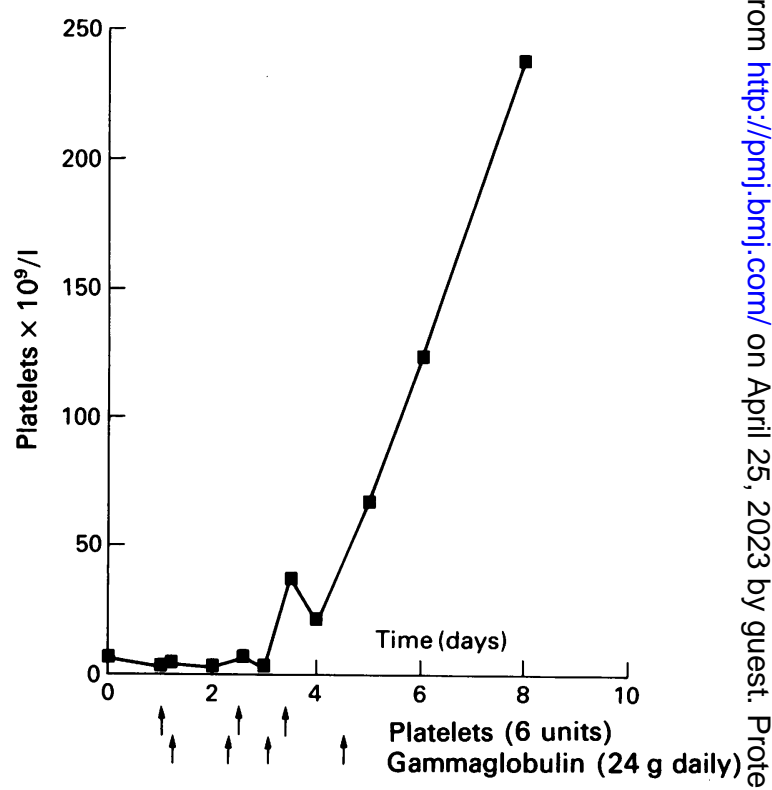

Figure 1 Increase in platelet count after treatment with intravenous gammaglobulin. 
immediately before and $60 \mathrm{~min}$ after platelet transfusions on days 1-4. Twice daily examination of optic fundi showed that retinal haemorrhages did not develop, and haematuria and other evidence of internal bleeding did not develop either. Within 4 days of starting treatment the platelet count increased to $60 \times 10^{9} / 1$ and the patient was allowed home. Subsequent platelet counts showed a complete recovery and further complications did not occur.

\section{Discussion}

Management of varicella-induced thrombocytopenia is difficult and deaths due to intracranial haemorrhage have been described. Platelet transfusion alone is ineffective because of rapid destruction by platelet auto-antibodies, ${ }^{2}$ or by other

\section{References}

1. Cohn, J. Thrombocytopaenia in childhood: an evaluation of 433 patients. Scand J Haematol 1976, 16: 226-240.

2. Feusner, J.H., Slichter, S.J. \& Harker, L.A. Mechanisms of thrombocytopaenia in varicella. Am J Hematol 1979, 7: 255-264.

3. Jerushalmy, Z., Kohn, A. \& DeVries, A. Interaction of myxoviruses with human blood platelets in vitro. Proc Soc Exp Biol Med 1961, 106: 262-269. mechanisms of platelet inactivation, lysis or desialization. ${ }^{3}$ Treatment with corticosteroids might potentiate the viraemia with fatal results, ${ }^{4}$ although in this case the haemorrhagic development was not obvious until 8 days after the initial rash appeared. Within $48 \mathrm{~h}$ of commencement of gammaglobulin, platelet counts started to increase, suggesting that inhibition of platelet destruction was occurring in response to treatment. After 3-4 days release of endogenous platelets into the circulation and their survival was suggested by the rapidly rising platelet count and the appearance of individual platelets on fresh blood films.

Intravenous gammaglobulin has a role in the management of immune-thrombocytopenia in several clinic settings ${ }^{5}$ and may also be of value in the treatment of the thrombocytopenia of haemorrhagic varicella infection.

4. Haggerty, R.Y. \& Eley, R.C. Varicella and cortisone. Pedia trics 1956, 18: 160-162.

5. Newland, A.C. The use and mechanisms of action of intravenous immunoglobulin: an update. Br J Haematol 1989, 72 $301-305$. 